\title{
Diseño de un modelo web intuitivo para una escuela de educación superior
}

\section{Design of an intuitive web model for a school of higher education}

\author{
PERALTA-SERRATO, Edgar†, GONZÁLEZ-RAMÍREZ, Claudia Teresa*, COLÍN-MORALES, José \\ Manuel y DELGADO-PICHARDO, Mauricio
}

\section{Tecnológico Nacional de México/Instituto Tecnológico de Zitácuaro, México}

ID 1 ${ }^{\text {er }}$ Autor: Edgar, Peralta-Serrato / ORC ID: 0000-0002-5785-5136

ID $1^{\text {er }}$ Coautor: Claudia Teresa, González-Ramírez / ORC ID: 0000-0002-4106-4583, Researcher ID Thomson: G-63132019, Open ID: 102182046108513149954

ID $2^{\text {do }}$ Coautor: José Manuel, Colín-Morales / ORC ID: 0000-0002-5821-3573

ID $3^{\text {er }}$ Coautor: Mauricio, Delgado-Pichardo / ORC ID: 0000-0002-9438-5217

DOI: $10.35429 /$ JHS.2020.11.4.22.33

Recibido Enero 20, 2020; Aceptado Junio 30, 2020

\section{Resumen}

En la actualidad nos encontramos en la Sociedad de la Información y Comunicación y las páginas web en las instituciones de Educación Superior representan un escaparate impresionante ante el mundo, ya que no podemos decir que es solo en su entorno, a través de estas plataformas web se informan las personas de los servicios y/o productos que se realizan en cada uno de éstas escuelas. Este trabajo presenta la aplicación de herramientas de un desarrollo web que facilitan su realización e implementación dando la oportunidad al experto en tener alternativas suficientes para llevarlas add hoc a una escuela. Proporcionando el alcance de proyección para estar en vanguardia y competitividad. Las nuevas tecnologías actualmente nos abren la posibilidad de mejorar el control de las tareas que son requeridas por el usuario. En este caso se busca diseñar un modelo web intuitiva e integral para propiciar la proyección da la institución de educación superior, en el Oriente de Michoacán

Web, Intuitiva, Integral, Vanguardia, Competitividad

\begin{abstract}
Currently we are in the Information and Communication Society and the web pages in Higher Education institutions represent an impressive showcase to the world, since we cannot say that it is only in their environment, through these web platforms people are informed of the services that are made in each of these schools. This work presents the application of web development tools that facilitate its realization and implementation, giving the expert the opportunity to have enough alternatives to take them ad hoc to school. Providing the scope of projection to be at the fore front and competitiveness. New technologies currently open up the possibility of improving the control of tasks that are required by the user. In this case, the aim is to design an intuitive and comprehensive web model to promote the projection of the institution of higher education, in the East of Michoacán
\end{abstract}

Web, Intuitive, Integral, Competitive, Vanguard

Citación: PERALTA-SERRATO, Edgar, GONZÁLEZ-RAMÍREZ, Claudia Teresa, COLÍN-MORALES, José Manuel y DELGADO-PICHARDO, Mauricio. Diseño de un modelo web intuitivo para una escuela de educación superior. Revista de Educación Superior. 2020. 4-11:22-33.

\footnotetext{
*Correspondencia al Autor (Correo Electrónico: claudia.lic@gmail.com)

$\dagger$ Investigador contribuyendo como primer autor.
} 


\section{Introducción}

Con la llegada de las nuevas tecnologías, las instituciones se encuentran en búsqueda de nuevos mecanismos que permitan agilizar el intercambio de información y actualizaciones en su desempeño laboral, tendiendo a acortar esa brecha tecnológica que existe entre docentes, padres, representantes, alumnos, etc. No obstante, aún se pueden encontrar muchas instituciones tanto recientes como experimentadas que no cuentan con las herramientas necesarias para mejorar tanto su eficacia como su eficiencia en este rubro. (Reyes Mauricio, 2020)

Es por esto que en una escuela de educación superior a través de un análisis riguroso se identifican las necesidades de sus departamentos, enfocándose principalmente en el de control escolar, área que posee un mayor flujo de datos y de carga de trabajo, siendo esto una gran problemática en la Institución, pero también se vislumbra la oportunidad de difusión a la institución aprovechando los recursos destinados.

La Universidad Americana de Comercio e Informática A.C (UACI), tras más de 5 años en el mercado educativo cuenta con varios campus a lo largo de la entidad entre los que destacan Zitácuaro, Altamirano, Huetamo, etc. La Universidad Americana de Comercio e Informática A.C cuenta con las licenciaturas de enfermería, contabilidad, administración de empresas, diseño gráfico, Ingeniería en sistemas computacionales, y enseñanza del idioma ingles en las modalidades escolarizada, semiescolarizada y en línea.

Actualmente la institución no cuenta con un sitio web que le permita difundir y brindar los servicios que ofrece a la comunidad lo cual ha impedido que esta crezca impulsada por las nuevas tecnologías y restándole competitividad frente a las demás instituciones que laboran en el mismo rubro.

El diseño de un modelo de un sitio web para la institución, es una solución integral que permite al instituto propiciar una mejor comunicación interna y externa, así como lograr proyectarse en la región Oriente de Michoacán

\section{Justificación}

La Universidad Americana de Comercio e Informática tiende a generar soluciones generales que se distinguen por impulsar una cultura académica de excelencia que infunde en sus miembros el compromiso con la integridad a la construcción de una sociedad con espíritu de servicio. Esto requiere de la implementación de las mejores herramientas disponibles en la actualidad. Desde el enfoque de la comunicación, no cabe duda de que el sitio web de una institución, se convierte en una ventana informativa, necesaria para mostrar procesos y brindar servicios que se lleven a cabo en el interior de la organización, permitiendo estrechar mejor la comunicación traspasando las paredes en su proyección a la comunidad y convirtiéndose en un gestor de estímulos para la participación motivante y activa de cada uno de los miembros de la comunidad educativa.

Actualmente la institución no cuenta con un sitio web que le permita difundir y brindar los servicios que ofrece a la comunidad lo cual ha impedido que esta crezca impulsada por las nuevas tecnologías y restándole competitividad frente a las demás instituciones que laboran en el mismo rubro. En la institución mucha información no se encuentra actualizada y requiere hacer una depuración de esta, y causa la creación de reportes incompletos o con errores. Comunicación interna ágil, actualmente para obtener información entre departamentos el personal debe ir personalmente a solicitar información.

\section{Objetivo general}

Diseñar, desarrollar e implementar un modelo de sitio web para la Universidad Americana de Comercio e Informática con herramientas agiles que permita un mejor servicio a sus diferentes usuarios académicos, administrativos $\mathrm{y}$ estudiantes.

\section{Objetivos específicos}

- Crear una página web para interactuar con los docentes, las estudiantes y la comunidad educativa en general.

- Desarrollar módulos que permitas proporcionar información veraz de los diferentes servicios que ofrece la institución. 


\section{Hipótesis}

Una página web intuitiva contribuye a facilitar la gestión de procesos en una institución de educación superior pequeña dando la oportunidad de proyección a su entorno.

\section{Metodología}

El tipo de estudio que para este proyecto es enfocado al desarrollo tecnológico, debido a la naturaleza de la solución planteada, la cual apoya el área de competitividad educación superior; el estudio inicia mediante la comparación de algunas aplicaciones web de educación superior, observando su funcionalidad y contenido. Determinando a los implicados expertos para el levantamiento de requerimientos y testeo de la información, ya que por su posición de expertos en los procesos las necesidades detectadas serán más objetivas.

El tipo de estudio implementado para el proyecto será de tipo mixta (cualitativocuantitativo), de carácter cualitativo, al evidenciar las mejoras con respecto a los procesos que se utilizan en la UACI, con un grupo de alumnos, la calidad de los distintos servicios y la disponibilidad de estos como un factor importante para poder contrastar los beneficios de la aplicación web contra los procesos tradicionales que se realizaban. Así mismo tiene carácter cuantitativo, al indicar de acuerdo con una serie de encuestas realizadas la relación entre diferentes variables y evidenciar mejoras o problemas con base a los datos recopilados sobre la aplicación web, y de desarrollo tecnológico. Empero, la metodología a emplearse para el desarrollo de la aplicación web será metodología en cascada.

\section{Instrumentos y equipo}

Para el proceso de recolección y validación de los datos se utilizan principalmente dos técnicas, la encuesta y la entrevista.

Encuesta: Se desarrolla dos formatos de encuesta, de acuerdo con las necesidades específicas que tienen los alumnos y personal (incluyendo docentes), cada una orientada a validar distintos aspectos de la aplicación web. La segunda de igual forma tiene un nombre específico y son de opción múltiple, además, pueden dar sus opiniones respecto a la aplicación web.
Entrevista. Se efectúa la entrevista para dar claridad sobre algunos temas referentes a los procesos de la institución.

\section{Modelado}

\section{Modelo en Cascada}

Este se compone de diferentes etapas las cuales debido a su fácil uso. Este se compone principalmente de cinco etapas:

1. Análisis de los requisitos de software

\section{Diseño del Sistema} principales:

Esta etapa se enfoca en cuatro puntos

- La estructura de datos.

- La arquitectura del software.

- El detalle procedimental.

- Caracterización de la interfaz.

\section{Codificación o Desarrollo}

4. Prueba

\section{Mantenimiento}

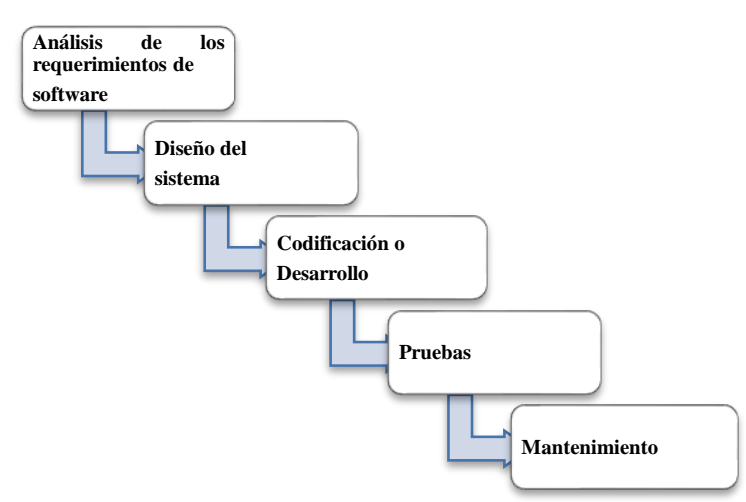

Figura 1 El modelo en cascada

\section{Análisis de los requerimientos}

A través de una investigación por los diferentes departamentos involucrados se determinaron diferentes aspectos que eran necesarios manejarse dentro del plantel, entre estos se encuentra la información sobre los estudiantes, materias y docentes. 
También se tomó en cuenta quién, y cómo se va a manejar esta información para obtener una mayor eficiencia durante su uso, por último, se consideró la forma en la cual se le va a presentar. Con la finalidad de definir los requisitos del software se subdividió esta tarea en cinco etapas las cuales se describen a continuación.

Reconocimiento del problema. Es esta etapa se analizaron las necesidades actuales del plantel, entre las cuales era el manejo actual de la información, la cual se realizaba por diferentes medios escritos y electrónicos causando duplicidad de esta, retrasos en procesos administrativos, principalmente de control escolar, también la falta de una plataforma mediante la cual los alumnos, docentes y la comunidad en general la puedan consultar.

Evaluación. Como su nombre lo indica se realiza una evaluación a partir de los problemas obtenidos, definiendo que funciones debe de realizar el software para dar solución. Tal como el concentrado de la información en una base de datos bien estructurada para evitar duplicados de esta, reportes de la misma según fue requerido por el personal del plantel, funciones que controlaran actividades del departamento de control escolar como son, registro, y administración de los datos de los alumnos, materias y docentes, registro de calificaciones, así como el reporte de las mismas en un formato tipo boleta el cual pueda ser impreso.

Modelado. En esta etapa se representa el comportamiento del software según las acciones del usuario, para esto se utiliza principalmente la herramienta de prototipos de aplicaciones web Balsamiq Mockups la cual permite crear modelos por medio de Wireframes (prototipos) interactivos.

Especificación. En esta etapa se clasifica los modelos en función de la información que manejan y los usuarios que tienen acceso a ella. Obteniendo el orden en el que se muestran las mismas en el sitio web y cuales tienen prioridad durante el desarrollo.
Revisión. Se revisa que todos los datos y funciones generadas en las etapas anteriores no tengan ambigüedad en la información, ni errores previos al diseño del sistema.

\section{Diseño}

Una vez definidos los requerimientos se realiza una estructura tentativa de la página principal usando diferentes herramientas como son el uso de programas de creación y edición de imágenes (Paint), herramientas de prototipos de aplicaciones web (Balsamiq Mockups), en el cual se estableció la estructura básica, así como los elementos que esta contiene. Sucesivamente con la estructura de la base de datos, usando el software administrador y Navicat, en la cual se definió las tablas necesarias para el funcionamiento del sitio (véase Figura 2).

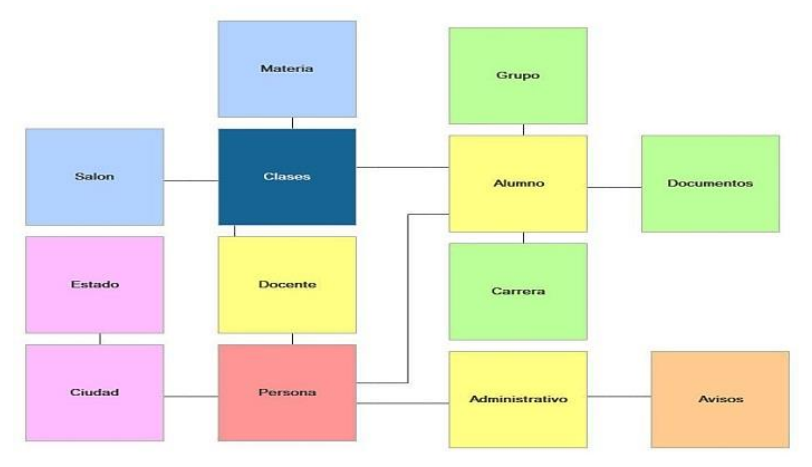

Figura 2 Borrador inicial de la base de datos del sitio web

Una vez definido el rol de los diferentes usuarios dentro del sitio web, se continuó con la estructura de los avisos y su ubicación como se observa en la siguiente Figura 3.

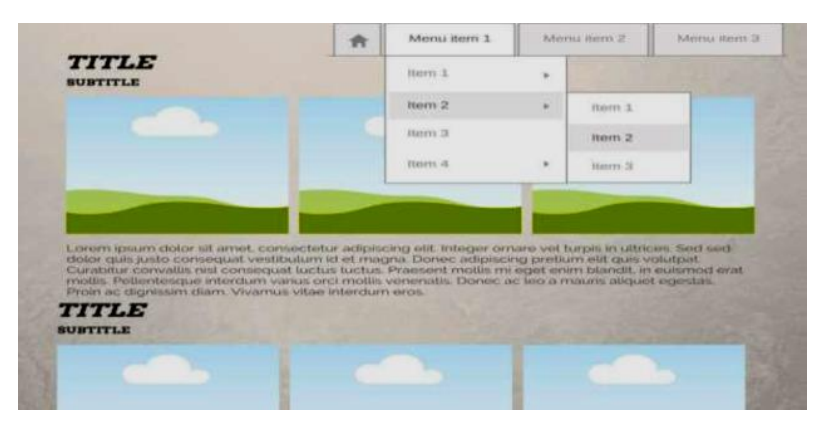

Figura 3 Borrador de la estructura de los avisos

\section{Desarrollo}

Una vez establecidos los requerimientos del sitio web, se continuo con el desarrollo de las diferentes pantallas que forman parte del sitio web, siguiendo una línea de diseño responsivo. 
El diseño responsivo es aquel que se adapta al tamaño del dispositivo desde el que se está visualizando la web, adaptando las dimensiones del contenido y mostrando los elementos de una forma ordenada y optimizada sea cual sea el soporte. El sitio web se desarrolló utilizando la programación AJAX, y el objeto XMLHttpRequest (XHR), la cual es una mezcla de cuatro tecnologías, JavaScript, XML, el objeto xmlHttpRequest y el Modelo de Objetos de Documento (DOM). Esto permite que el sitio web obtenga varias ventajas en su uso las cuales comprenden:

Actualización dinámica sin tener que recargar la página completa.

Reduce el ancho de banda usado al navegar dentro del sitio web.

Mejora la interacción con el sitio web.

\section{Programación}

Como Front-End se utiliza HTML5, PHP, JavaScript y dando estilo con a las pantallas con hojas de estilo (CSS) y JavaScript por medio de Bootstrap. En Back-End se desarrolla el módulo en PHP principalmente. Entre todas las funciones conocidas de JavaScript se menciona objetos xmlHttpRequest los cuales mediante el método citado a continuación envían datos a un servidor PHP.

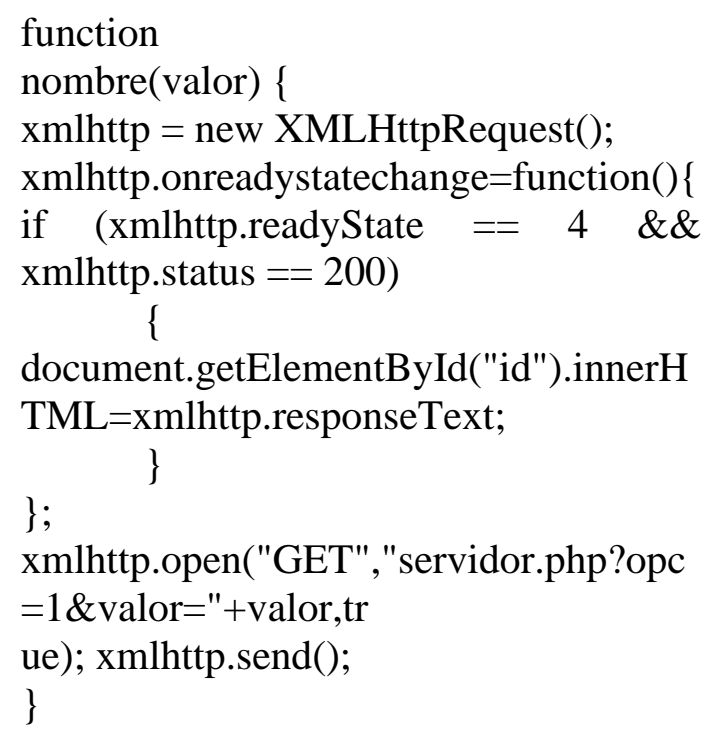

En el servidor PHP se establece la conexión al servidor de bases de datos para procesar la solicitud o el almacenamiento de la información enviada.
Esto se realiza orientado a objetos otorgando a la conexión mayor simplicidad y facilitando la depuración de errores. Únicamente cuando los datos han sido procesados en su totalidad, la función inserta el resultado en el elemento que se ha preseleccionado por medio del api DOM, usando el objeto "document", el cual en el ejemplo anterior apunta al identificador del elemento (id).

Como se describió anteriormente el sitio web al seguir un diseño responsivo requiere que el elemento donde se va a insertar los datos ya cuente con el código necesario para adaptarlo o bien que los datos que son recibidos tengan implementado dicho código. En este punto es cuando se hace uso del framework Bootstrap, el cual está basado sobre una estructura divida en 12 columnas las cuales se gestionan de manera que se obtenga el diseño deseado, según cuatro tamaños de pantalla.

\section{Teléfonos $(<768 \mathrm{px})$.}

Tabletas $(>=768 \mathrm{px})$.

Computadoras de escritorio (>=992px).

Computadoras de escritorio (>1200px).

Esto se logra mediante clases definidas previamente las cuales escalan el tamaño de la ventana de visualización. Normalmente los datos son recibidos en una caja con un diseño de recuadro el cual se le da la clase predefinida “. well" propia del framework, la cual se encontraba dentro de otro contenedor $\langle$ div $>$ el cual se le ha colocado la clase ". Container" para indicar que este es el contenedor principal donde se encontraran las columnas descritas anteriormente. Dentro de este se coloca otro contenedor el cual indica el tamaño el cual se va a escalar en los diferentes dispositivos mediante las clases explicadas anteriormente $<$ div class="col-md-12 col-sm-12 col-xs-12 well" style="margin-top: $\quad-20 \mathrm{px} ; "$ $\mathrm{id}=$ "contenido">

En el caso de los mensajes hacia el usuario, se muestran mediante el uso de JavaScript y Bootstrap, usando la clase ". modal" la cual viene predefinida y la que nos sirve para mandar mensajes más estilizados. 
Al mostrarse la información se hace uso de animaciones las cuales le dan un toque original al sitio web, para esto se utiliza la librería animate.js y su función “. animated” la cual tiene predefinida varios estilos de animaciones para el elemento donde se implemente.

\section{Base de datos}

El motor de bases de datos utilizado es MySQL. y como manejador de bases de datos es phpMyAdmin, el cual es la herramienta de administración de MySQL más utilizada en páginas web. El motor de bases de datos que es utilizado en los diferentes sitios de alojamiento de sitios web ya viene configurado por el proveedor por lo cual no hay necesidad de realizar instalaciones o configuraciones adicionales durante esta etapa del desarrollo.

\section{Funcionalidad}

El sitio web fue desarrollado pensando en obtener respuestas de una manera mucho más rápida y eficaz, permitiendo al usuario interactuar de manera sencilla, veloz e intuitiva, por este motivo se decidió crear una web totalmente visual, fácil de leer y comprender con diseño minimalista e intuitivo.

\section{Módulo de avisos}

Este módulo es especialmente para el uso de la dirección, quien se encarga de difundir los avisos a la comunidad al no existir un departamento específico para este fin, ver Figura 4, esto incluye avisos de actividades, información a la comunidad, avisos de ocasión, entre otros.

El diseño se dice intuitivo, porque el gestor al ver la pantalla se encontrará con diferentes botones que le ayuda a dar un mejor formato a los avisos que se publican, entre los que se encuentran: botón que se encarga de poner el texto en negritas, en cursiva, agregar un enlace en el texto, para centrar el texto, agregar saltos de línea y seleccionar uno o más archivos o imágenes para que se publiquen junto con la imagen.

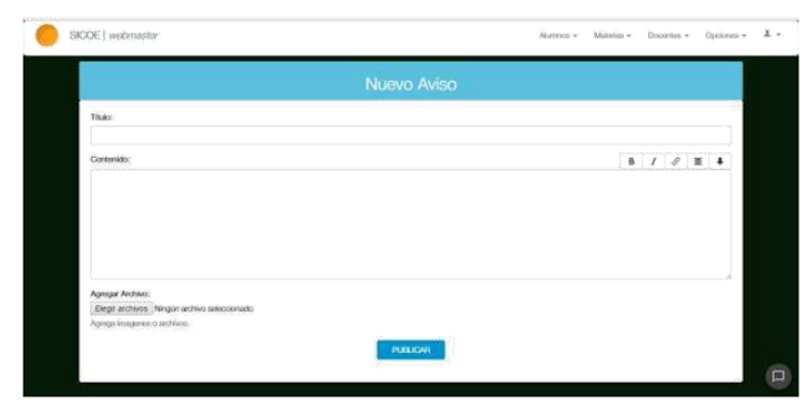

Figura 4 Pantalla para la publicación de nuevos avisos

\section{Módulo de control escolar}

Este módulo se encarga de realizar diferentes actividades dentro del sitio web las cuales son registro y actualización de datos de alumnos y de la documentación que se les solicita a los alumnos, registro y consulta e impresión de boletas de calificaciones, ver Figura 5.

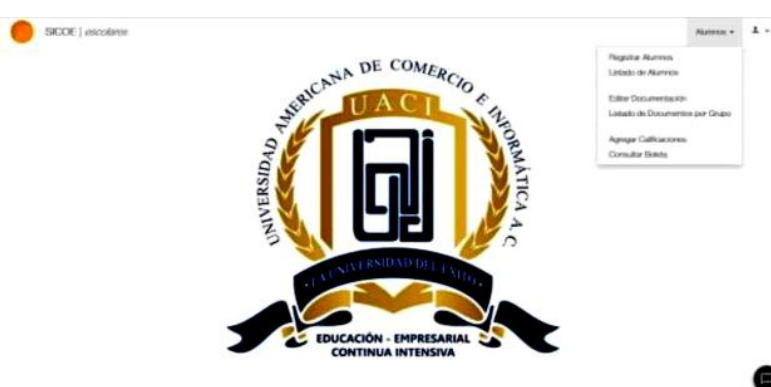

Figura 5 Pantalla de inicio para el departamento de control escolar

El registro de alumnos se realiza tomando en cuenta sus datos generales, para esto se utiliza el siguiente código el cual indica la codificación con la cual debe guardarse esta información.:

\section{\$con->consulta ("SET NAMES 'utf8'");}

Para listar a los alumnos se muestra un reporte de todos los inscritos en el plantel ordenados por carrera, las búsquedas en la base de datos son dinámicas, arrojando al alumno que se busca de manera fácil, por medio de su nombre o cualquiera de sus apellidos, o, un botón mediante el cual se puede filtrar los resultados, mostrándonos solo los alumnos inscritos a una carrera en específico, observar Figura 6 


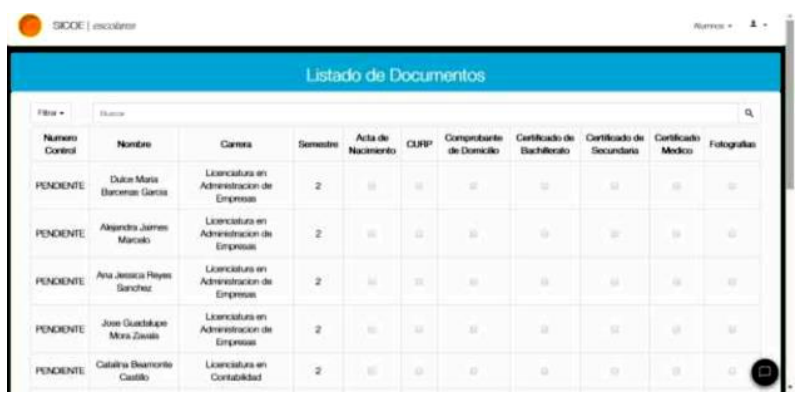

Figura 6 Pantalla de listado de documentos

Las para la administración de calificaciones cuentan con las opciones necesarias para realizar la actualización de estas. Asimismo, se puede imprimir la boleta de calificaciones por medio del botón habilitado para tal fin, ver la siguiente Figura 7.

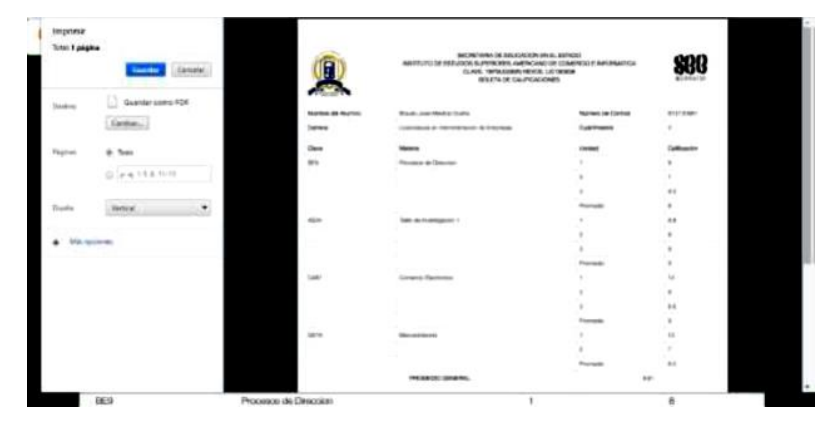

Figura 7 Pantalla de impresión de boletas

\section{Módulo de docencia}

En el departamento de docencia se maneja una extensión al módulo de control escolar, este cambio fue requerido debido a que ciertas actividades de control escolar fueron delegadas a dicho departamento para ofrecer mayor rapidez en el procesamiento de estas, enfocándose en la administración relacionada con las materias y los docentes por lo tanto cuenta con un menú dedicado a cada uno de estos apartados. Las pantallas especiales para materias (ver Figura 8).

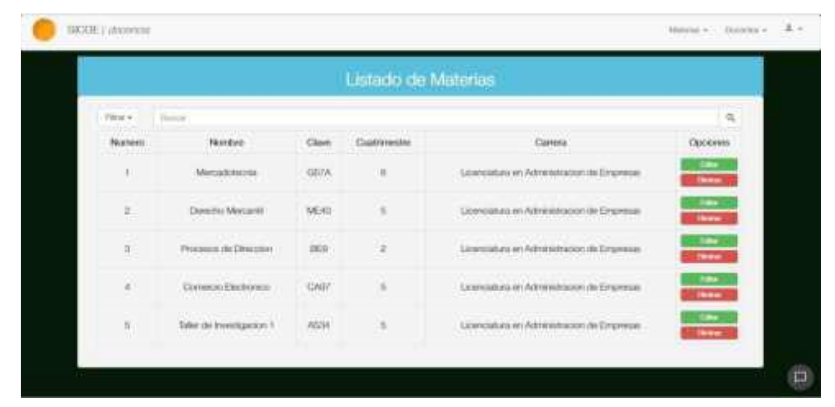

Figura 8 Pantalla para la gestión de materias
Como es habitual en el diseño se agregó una barra de búsqueda dinámica, la cual como se puede observar se incrusto como parte de la plantilla destinada a reportes de información para facilitar la localización de esta dentro de grandes bloques. La edición de las materias se habilito para los casos en que los planes de estudios cambien y sea necesario moverlas del semestre, esto es de suma importancia a la hora de realizar horarios o consultar boletas de calificaciones ya que el sistema ordena en ambos casos la información en base al semestre y carrera que este registrado. También se desarrolló una interfaz especial para la carga de horarios (Figura 9) en la cual como se muestra la selección al docente que se asignará un horario y aparecerá una tabla marcado las horas y días laborables.

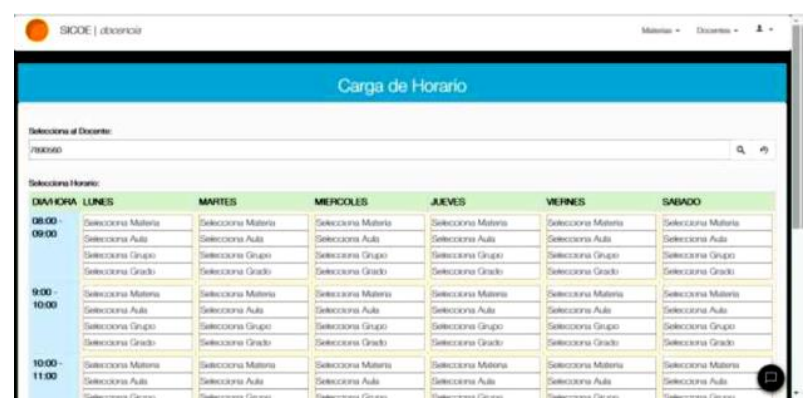

Figura 9 Pantalla para la carga de horarios

En dicha tabla tendrá por cada día/hora cuatro selectores con los cuales se podrá seleccionar entre las materias disponibles, aquella que el docente impartirá, también se puede seleccionar el aula donde se llevará a cabo la clase, así como el grupo al que será impartida. La edición de horarios (Figura 10) es similar a la de carga de horario con la finalidad de que al usuario se le facilite la identificación de los campos que debe de actualizar.

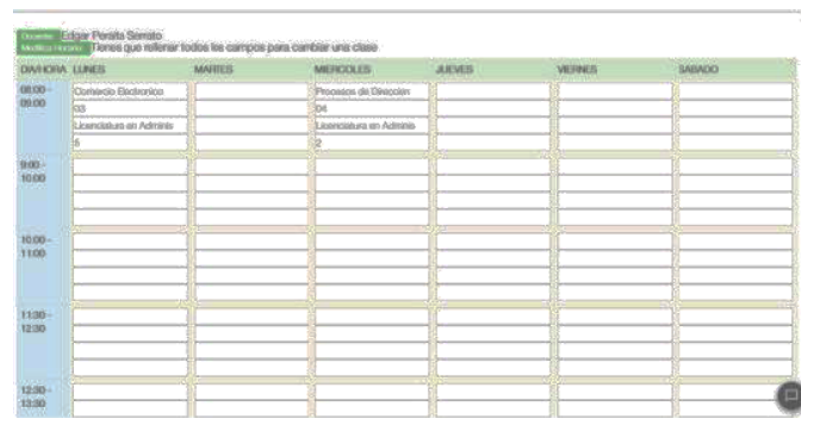

Figura 10 Pantalla para la edición de horarios 


\section{Pruebas}

Se realizan pruebas periódicas para corregir errores que se generen durante el desarrollo de la página web (información no deseada en pantalla) y se hacen cambios pertinentes para que el sitio web funcione correctamente.

Implementación del sitio web Una vez terminado del sitio web, se realiza una amplia investigación acerca de los servidores web, sus costos y lenguajes soportados con la finalidad de escoger el que más se adecuara a los requerimientos del sistema y que además fuera accesible para el usuario. La implementación del sitio web consiste en varias etapas ya que durante algunas de ellas se requería la introducción de algunas funcionalidades extra que son primordiales para solventar algunos de los problemas descritos anteriormente.

Etapas en la implementación del sitio web de la universidad:

- Exportación de ficheros en el servidor web.

Exportación bases de datos al servidor de bases de datos.

Implementación del sistema de comunicación en tiempo real (chat).

4) Implementación de un blog académico solicitado por la institución para la publicación de artículos educativos e investigación.

Generación de respaldos para el sitio web.

El hosting cuenta con la herramienta phpMyAdmin la cual es muy efectiva en el manejo de bases de datos MySQL, por este motivo se crea un respaldo completo de la base de datos en formato SQL el cual se importa desde el mismo hosting siguiendo los siguientes pasos:

Se accede a la sección de bases de datos del hosting por medio del menú superior en el sitio web.

Una vez hecho esto se da clic en el botón de agregar una base de datos y se asignó un nombre (ver Figura 11).

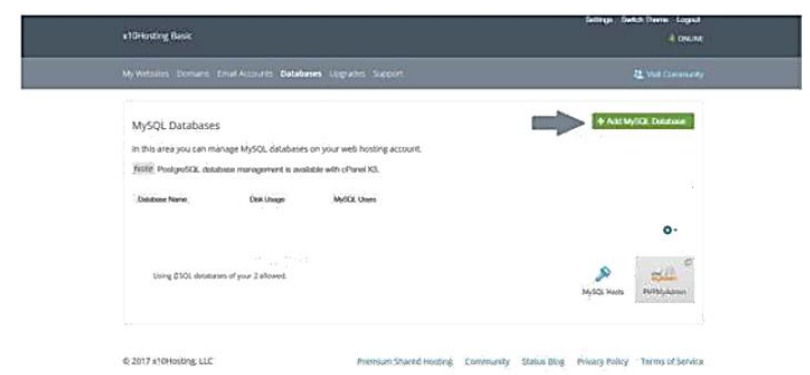

Figura 11 Pantalla del servidor de bases de datos donde se nos indica donde pulsar para agregar una base de datos nueva

Una vez hecho esto se realiza la importación, de la base de datos usando phpMyAdmin alojado dentro del mismo servidor web, de esta manera se evita tener que instalar alguna manejador de bases de datos en la computadora por la cual se realiza la exportación de la base de datos.

Implementación del sistema de comunicación en tiempo real (chat). Para la implementación de un chat durante la etapa de diseño se declara utilizar un servicio externo ya que estos poseen diversas características embebidas que nos ayudan minimizar los tiempos de desarrollo. Después de analizar los diferentes servicios disponibles en la red, se optó por implementar la propuesta de ChatWee no solo por su facilidad de instalación y personalización, sino además por su gestión. Para esto se crea una cuenta nueva la cual va ligada al correo electrónico del plantel que es donde se reciben las notificaciones referentes al chat, el diseño del chat es de acuerdo al que posee el sitio web, se le coloco un título y posteriormente se insertó una pequeña línea de código inmediatamente después del código del encabezado del sitio web para que este comenzara a ejecutarse tan pronto se terminaba de cargar el sitio web (Figura 12).

<scriptsrc="https://chatweeapi.com/v2/script/59 1a6eb2bd616dfb1baxxxxx.js" $></$ script $>$

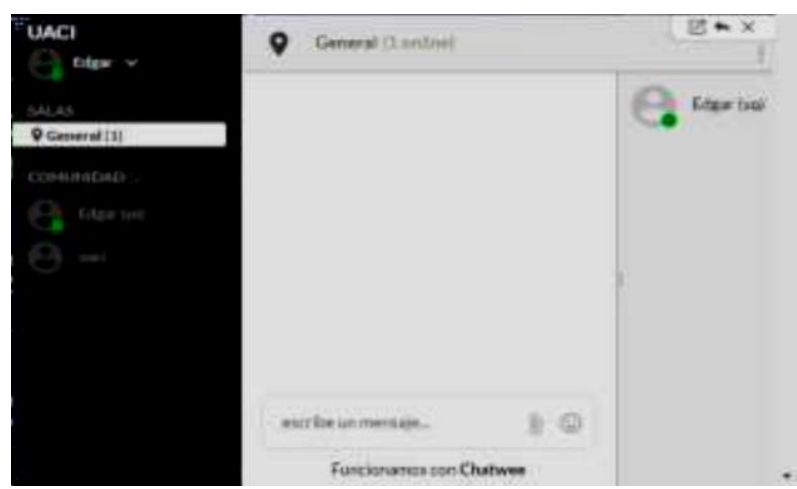

Figura 12 Pantalla principal del chat

PERALTA-SERRATO, Edgar, GONZÁLEZ-RAMÍREZ, Claudia Teresa, COLÍN-MORALES, José Manuel y DELGADO-PICHARDO, Mauricio. Diseño de un modelo web intuitivo para una escuela de educación superior. Revista de Educación Superior. 2020 
Con la implementación de este servicio se solventan dos problemas: la comunicación interna entre el personal de la UACI, necesaria para evitar pérdidas de tiempo y la comunicación del personal con cualquier visitante de la página que requiera informes acerca de los servicios que el plantel ofrece, así como el envío de archivos internamente.

Implementación de un blog académico solicitado por la institución para la publicación de artículos educativos e investigación mediante la petición directa del rector de la Universidad Americana de Comercio e Informática, donde los docentes podrán publicar artículos académicos o de investigación referentes a las materias que estos impartan dentro del plantel, con la finalidad de brindar un mejor servicio a la comunidad estudiantil ampliando sus fuentes de consulta de información. Para esta tarea se hizo uso del correo electrónico del plantel la cual pertenece a la compañía Google, esto nos trajo una ventaja la cual es que dicha compañía ofrece la posibilidad de crear un blog mediante su servicio Blogger (Figura 13 y 14).
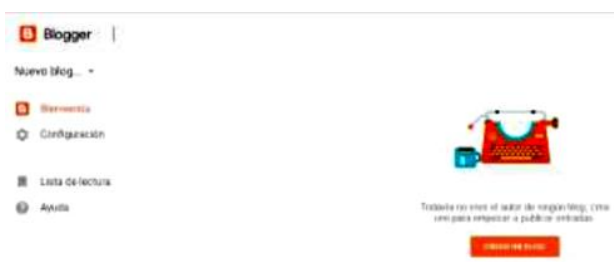

Figura 13 Pantalla de inicio de Blogger

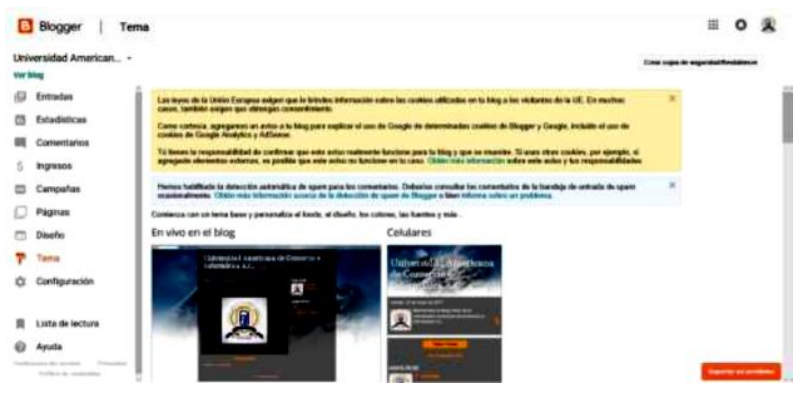

Figura 14 Pantalla de personalización del blog

En el sitio web se agregaron los botones con los vínculos a las diferentes redes sociales del plantel los cuales fueron colocados en una barra lateral flotante mediante el uso de CSS ver Figura 15.

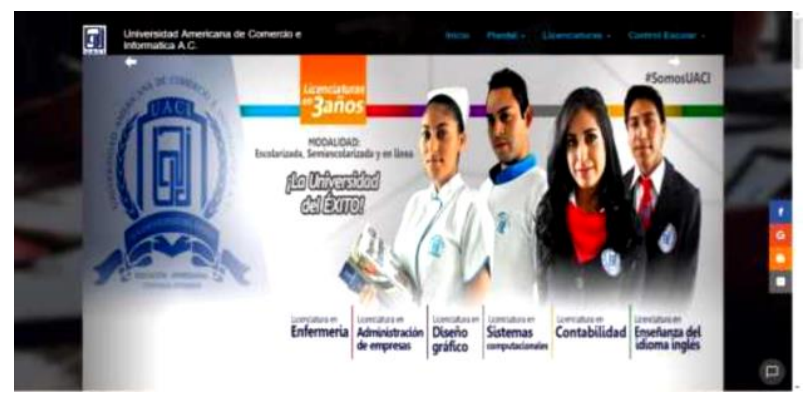

Figura 15 Pantalla de inicio del sitio con los botones sociales implementados

\section{Generación de respaldos para el sitio web}

Una de las funciones principales del sitio web es la administración de información principalmente las actividades que se realizan en control escolar, estos datos son de suma importancia para la UACI. Es por eso que se necesita generar respaldos de toda esta información de manera automática y periódica sin que el personal se tenga que preocupar por esto y manteniendo los datos seguros ante cualquier imprevisto. Para ello se recurrió al servicio de respaldos que nos ofrece el mismo hosting.

\section{Resultados}

Posteriormente a la implementación del sitio web, se obtuvieron resultados importantes para mejorar la imagen corporativa del plantel, así como también incrementar la eficiencia en el desarrollo de las actividades de control escolar descritas con anterioridad, disminuyendo el tiempo, esfuerzo requerido para realizarlas y generando un ahorro de diseño en el gasto de recursos materiales. También se realizó una encuesta al personal, estudiantes, docentes de UACI, y personas que asistían a la universidad, de los cuales el $85 \%$ expreso su opinión:

¿Cómo considera el diseño del sitio web de la Universidad Americana de Comercio e Informática A.C.?

\section{Diseño de Sitio Web}

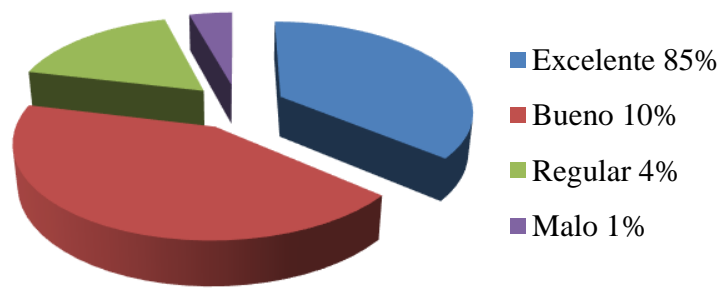

Gráfico 1 Porcentaje de aprobación del diseño del sitio web

Fuente: Elaboración propia

PERALTA-SERRATO, Edgar, GONZÁLEZ-RAMÍREZ, Claudia Teresa, COLÍN-MORALES, José Manuel y DELGADO-PICHARDO, Mauricio. Diseño de un modelo web intuitivo para una escuela de educación superior. Revista de Educación Superior. 2020 
¿Cómo considera el tiempo de respuesta al consultar información?

\section{Tiempo de respuesta}

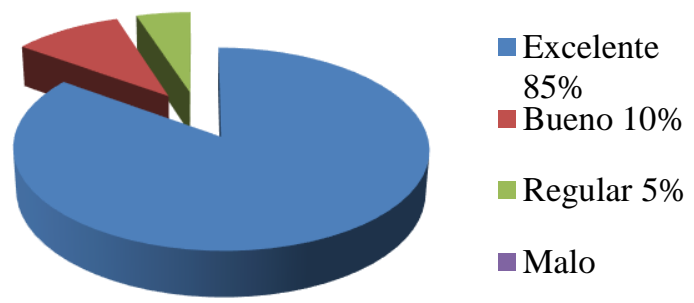

Gráfico 2 Tiempo de respuesta

Fuente: Elaboración propia

¿Cuánto tiempo era necesario para obtener boletas de calificaciones y cuánto se necesita actualmente?

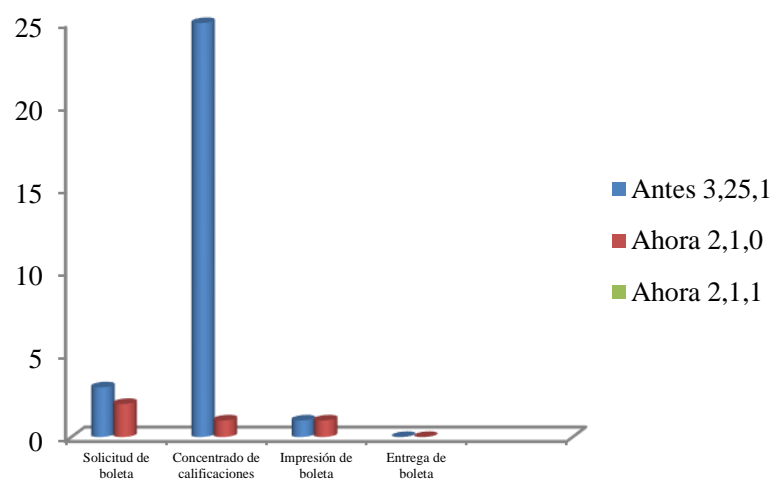

Gráfico 3 Tiempo de obtención de boletas por procesos Fuente: Elaboración propia

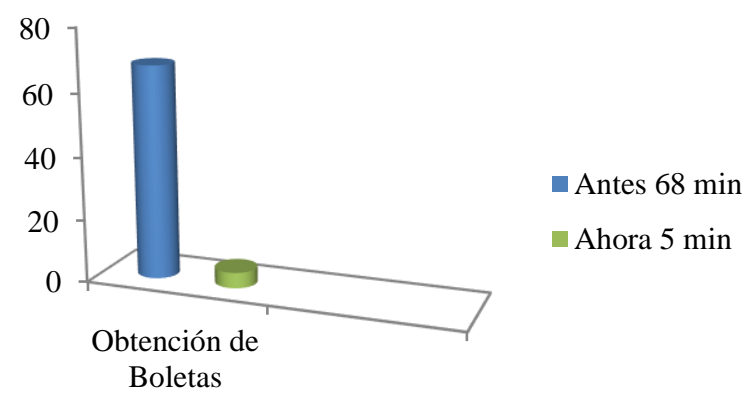

Gráfico 4 Tiempo de obtención de boletas Fuente: Elaboración propia

De lo anterior podemos observar que el ahorro de tiempo durante la obtención de boletas es de un $92.65 \%$, si no tomamos en cuenta factores como que el encargado de control escolar tenga que salir a alguna diligencia o reunión y esto aumente el tiempo de espera en la entrega de las boletas, mejorando aun el porcentaje de ahorro de tiempo.
¿Qué tan completa encuentras la información que presenta el sitio web acerca de la UACI?

\section{Diseño de Sitio Web}

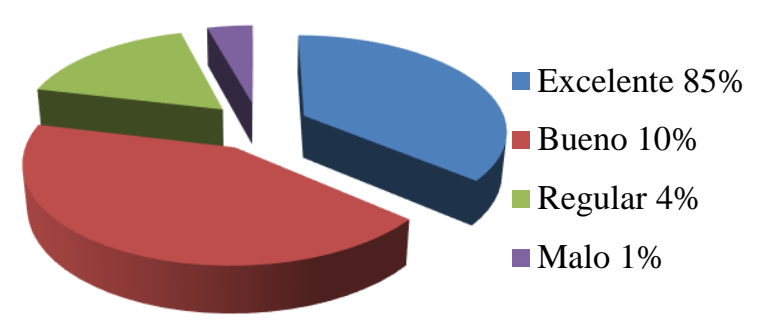

Gráfico 5 Contenido del Sitio Web

Fuente: Elaboración propia

Resultados cuantitativos.

- Se redujo la redundancia de datos en un $100 \%$.

- Se aumentó la eficiencia del manejo de información.

- La consulta de información es un $90 \%$ más rápida.

- Se disminuyó el número de personas solicitando información directamente en los departamentos de control escolar y docencia.

Resultados Cualitativos.

- $\quad$ El diseño del sitio web es intuitivo.

- Se puede consultar la información desde diferentes dispositivos con distintas resoluciones.

- El sitio ofrece información de manera eficiente.

- Se le otorgo mayor competitividad a la Universidad Americana de Comercio e Informática A.C., en el uso de la información, una posición relativa en el mercado de educación en la zona oriente de Michoacán y sobre todo estableciendo relación con sus clientes potenciales y usuarios. 


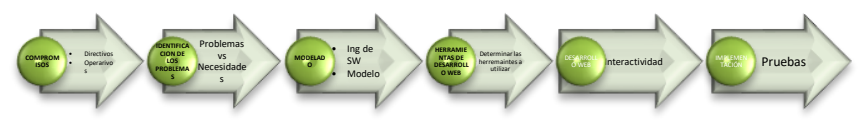

Figura 16 Propuesta de Modelo para web intuitivas Fuente: Elaboración propia

En la Figura 16 anterior se propone un modelo práctico para llevar a cabo de manera ágil el desarrollo de una página web intuitiva, sirviendo éste como referencia

\section{Conclusiones}

Se ha comprobado que el impacto de tener una plataforma web depende de la relevancia que las organizaciones decidan dar a este aspecto (Honiges, 2013). El desarrollo eficiente de la web depende del grado de compromiso en las personas de la institución y de la contribución veraz en información que den, no de su contexto geográfico o su titularidad, como también corroboraron las entrevistas realizadas, al poner de manifiesto situaciones de responsabilidad heterogéneas por parte de las comunidades educativas (Álvarez e Inés, 2017). En este trabajo si manifestaron el compromiso de la realización e implementación del trabajo integral web en la UACI, siendo un estímulo el diseño y desarrollo de la web (RodríguezMiranda, PozuelosEstrada y León-Jariego, 2014).

Dado que estamos en la Sociedad de la Información y la Comunicación, las escuelas de educación superior no deben descuidar su imagen en la red. En ésta misma hay infinidad de herramientas para construir prototipos de plataforma web, desde programas profesionales hasta pequeñas aplicaciones web que nos permiten añadir enlaces a nuestros diseños para hacerlos navegables, siendo el caso de Balsamiq - Moqups, herramienta que facilita su aplicación y facilitando su diseño e implementación en una organización como UACI, dando excelentes resultados como se han mostrado.

Podemos decir que en verdad una página web intuitiva contribuye a facilitar la gestión de procesos en una institución de educación superior pequeña dando la oportunidad de proyección a su entorno.
El modelo es una propuesta entre tantas que hay en la red, la difencia radica en que con los elementos de la ingeniería de software, herramientas de desarrollo web y las consideraciones de la organización se puede hacer un modelo o esquema a seguir facilitando todas las fases, al encargado de TI en las organizaciones, teniendo la capacidad de adaptarlo a su shell, esquema y modelo de trabajo enriqueciendo.

Esto contribuye a la sustentación para gestión de la educación del siglo XXI: una educación transformadora que rompa con el mito del estudiante promedio y del currículo rígido, y contemple el diseño universal para el aprendizaje (DUA) como el paradigma que ofrece oportunidades de aprendizaje a todas las personas. (Carmona, 2020).

\section{Discusión}

Hoy en día es increíble el número de personas que tiene un dispositivo electrónico y el uso que le da, En ese entendido tener una web se ha convertido en algo indispensable para toda organización. Las nuevas generaciones y la época que se vive optan cada vez más por buscar y elegir el producto y/o servicio en las webs por las comodidades que ofrece. Es fundamental para una organización disponer de un punto virtual donde dar a conocer sus servicios a clientes potenciales y situarse en la competitividad que se encuentran las instituciones hoy en día, y si la plataforma web es intuitiva e inteligente-aplicando tecnología puntual- le da a la organización ventaja sobre las demás. No es requisito actualmente ser una institución grande, con un gran número de estudiantes para tener una aplicación web, lo que sí es requisito es contar con una aplicación web que proporcione la visualización de la organización y de información atractiva, útil interesante, capaz de motivar a seguir visitando la página.

\section{Referencias}

Álvarez, C. e Inés, J. (2017). The management of schools' websites in Cantabria, Spain. Research in Learning Technology, 25(1), 1270579.

doi: https://doi.org/10.1080/21567069.2017.127057 9 
Johnson, D. (2010). Computing in the clouds. Learning \& Leading.ISTE. Vol. 37, No 4, Dic Ene 2009-2010

De Miguel, Santa María Adoración y Plattini, Mario. Concepción y Diseño de Base de datos Del modelo E-R al modelo relacional. Ed. Addison Wesley Iberoamericana, R.

García-Romero, J. E. y Faba-Pérez, C. (2015). Desarrollo e implementación de un modelo de características o indicadores de calidad para evaluar los blogs de bibliotecas escolares de centros de educación infantil y primaria. Revista Española de Documentación Científica, 38(1), 67-78. doi: https://doi.org/10.3989/redc.2015.1.1169

Hartshorne, R., Friedman, A., Algozzine, B., Isibor, T. (2006). Secondary schools online: Are high school web sites effective? American Secondary Education, 34(2), 50-66.

Honiges, A. (2013). School image in the context of new communication technology. Knowledge Horizons-Economics, 5(2), 162166. Hu, C. y Soong,

Joyanes Aguilar, Luis. Programación en Java 2, Algoritmos, Estructura de Datos y Programación Orientada a Objetos. Ed. Prentice Hall.

López Quijado, José. Domine PHP y MySQL. 1ra. edición. Ed. Alfaomega.

Mark Otto, Jacob Thornton, Chris Rebert. (2011). Bootstrap. 10/02/2017, de founding team Recuperado: http://getbootstrap.com/

MarketiWeb (2020) Diseño de páginas Web. Recuperado: http://www.marketiweb.com

Merca20.com(2020) 3 Aspectos para Analizar la Información y Emplearla.Recuperado: https://www.merca20.com/3-aspectos-paraanalizar-la-informacion-y-emplearla/

Mysql Manuales de Referencia del Manejador de Base de Datos. -Disponible en Interneten:https://dev.mysql.com/doc/refman/5. $5 / \mathrm{en} /$.

Pressman, Roger S. (2005). Ingeniería del Software. Ed. McGraw-Hill. Sexta edición. México
Rancing N.P.(2020). Microlearning en Educación Superior.Universiada Oberta de Cataluña. Master en Educación y TIC.Buenos Aires, Argentina

Rodríguez-Miranda, F. P., Pozuelos-Estrada, F. J. y León-Jariego, J. C. (2014). The role of ICT coordinator. Priority and time dedicated to professional functions. Computers \&Education, 72, 262-270. doi: https://doi.org/10.1016/j.compedu.2013.11.009

Steiman, J. (2020). Las prácticas de enseñanza: -en análisis desde una Didáctica reflexiva-. Miño y Dávila.

Sommerville, Ian. (2002). Ingeniería de software 6ta edición. Prentice-Hall.

Stewart, T., Jacob, A. M., Jensen, L. I. (2012). School site visits: What can we learn from choice schools in Milwaukee. Recuperado de http://files.eric.ed.gov/fulltext/ED530069.pdf

Tobias Ratschiller, Marc Delisle. (2012). phpMyAdmin's documentation. 18/04/2017, de The phpMyAdmin devel team Sitio web: https://docs.phpmyadmin.net/en/latest/

Wells, J. A. y Barron, A. E. (2006). School web sites: Are they accessible to all? Journal of Special Education Technology, 21(3), 23-30. doi:

https://doi.org/10.1177/016264340602100303

w3schools.com. (2015). Bootstrap 3 Tutorial. 25/01/2017, de w3schools Sitio web: https://www.w3schools.com/bootstrap/default.a sp. 